\title{
Ideologie der Sesshaftigkeit und Mobilität
}

\section{Danielle Bazzi (Zürich)}

Zusammenfassung: Es stellt sich die Frage, wie unsere Psyche mit einer sich zunehmend dynamisierenden Umwelt interagiert. Ich umkreise den Begriff «Mobilität» aus mentalitätsgeschichtlichen, ethnologischen und psychoanalytischen Perspektiven, um zu zeigen, dass sowohl Alltags- wie Wissenschaftskonzepte unsere Einstellung dem Phänomen gegenüber beeinflussen. Ich stelle die Annahme, dass sich die Psyche einerseits in linearen Entwicklungsprozessen bilde und andererseits durch die Realität zur Anpassung genötigt werde, in Frage, weil ich denke, dass dadurch die Wahrnehmung eines psychischen Funktionierens blockiert wird, das an einer nichtlinearen und kontinuierlichen Schaffung von neuen bewussten und unbewussten psychischen Elementen beteiligt ist. Ich vertrete den Ansatz eines relationalen, wechselseitigen Zueinandersetzens von äusseren und inneren Kräften und möchte dies in Absetzung zu dem zeigen, was ich «Ideologie der Sesshaftigkeit» nenne. Im «Essay sur les variations saisonnières des sociétés eskimo" von Marcel Mauss und dem Konzept der Oszillation von $P-S \Leftrightarrow D$ von Wilfried Bion finde ich Beschreibungen von sozialen und psychischen Funktionsweisen, die mein Anliegen erhellen. Miteinander in Beziehung gesetzt, fordern sie hergebrachte Definitionen von Unbewusstem und Empirie heraus. Ebenso kommen gängige Abgrenzungen von Individuum und Gesellschaft in Bewegung, wenn - wie angenommen - fortdauernde psychische Funktionsweisen es ermöglichen, sowohl subjektiven wie sozialen Empfindungen ständig neuen Sinn zu geben.

Schlüsselwörter: Oszillation von $\mathrm{P}-\mathrm{S} \Leftrightarrow \mathrm{D}$, Bion, Ethnologie, Migration

Haben wir ein Problem mit der Mobilität, liegt der Gedanke nahe, dass uns bei der Vorstellung etwas überfordert, dass wir sie nicht wirklich denken können. Was bewirkt der Verlust des Standortes, wie verfahren wir in Bewegung mit neu eingehenden Informationen? Ist uns das Ungewisse, das bei Standortwechseln und deren Übergang eintrifft, unheimlich? - Ich meine, dass die Forderung nach Innehalten nicht helfen kann. Wir handeln uns Leid ein durch eine Ideologie, die besagt, dass sich das «Normale», das «Gesunde», das «Reife» nur linear in einer 
«beständigen» Verankerung an einem Ort entwickeln kann. Die Entstehung dieser Ideologie halte ich für ein lokales Artefakt.

Analysand: Gestern beim Heimfahren mit dem Zug dachte ich, es ist immer alles so gleich - ein paar Baustellen hat es -

Analytikerin: Neue Gebäude entstehen

Und am Bahnhof hat es so Bretter und Hinweise, wo jetzt die Busse stehen. Aber wenn ich fremd wäre, ich weiss nicht, ob ich die finden würde

Etwas durcheinander

Ich finde, man muss so kanalisiert gehen (mit Enttäuschung in der Stimme)

Das bedauern Sie

Ja, das hat mir schon leid getan. Das ist wohl für die, die in den Büros arbeiten und aufEffizienz geschult werden. - (Lacht so in sich hinein) Und dann haben die den Fussgängerstreifen abgekratzt, man sieht nur noch ein wenig vom Gelb - und so Füsse hingemalt, so grüne, so Sohlen; kleine, zwei aufeiner Seite und zwei auf der anderen Seite.

Ein Analysand spricht kurz vor dem Ende der Stunde und oszilliert zwischen Gefühlen der Unfertigkeit, von sich nicht zurechtfinden (PS) und Enttäuschung über die Orientierungsvorgabe (D). Im Augenblick, wo das Durcheinander, das Gefühl beim bevorstehenden Verlassen des Settings ausgesprochen ist, taucht blitzschnell die Ordnung auf und wird als einengend empfunden. Dann freuen die Fuss-Piktogramme auf der einen und der anderen Seite des Übergangs, während die geometrischen Zeichen verblassen.

Meine folgenden Überlegungen möchte ich als Arbeit an einer möglichen Interpretation von «Mobilität» unter dem Aspekt der psychischen Anforderungen betrachten. Mobilität ist einerseits Bewegungslust, andererseits kann sie Fragmentierungsangst oder Verlustangst berühren. Es interessiert mich, welche Auswirkung unterschiedliche theoretische Konstrukte auf unser Erleben haben. 
Wird die Mobilität nicht als etwas Gegebenes, sondern als Ausnahme empfunden, erhält die Sesshaftigkeit etwas Ideologisches. Könnte im Ideal der Stabilität nicht eine reaktive Wendung gegen die Arbeitsanforderung der Oszillation von $\mathrm{P}-\mathrm{S} \Leftrightarrow \mathrm{D}$ stecken?

Melanie Klein zufolge ist die paranoid-schizoide Phase PS eine Entwicklungsstufe. Aufgrund der Angst vor einer angreifenden Mutter wird der Sadismus ins Objekt «herausgeworfen», um gleichzeitig sich zu schützen und das Objekt zu vernichten. Diesen expulsiven Mechanismus versteht sie als erste Abwehrform und frühen Vorläufer der Symbolisierungsfunktion. Die darauffolgende depressive Phase D gilt für Klein als die zentrale Entwicklungsstufe des Kindes. Die sichere Internalisierung des guten Objekts erfolgt, wenn als Konsequenz von Hass und Aggression der Verlust und das Betrauern der Brust der Integrität des Objekts Platz macht (Klein, 1975; 1996).

Sesshaftigkeit hat mit politischer und wirtschaftlicher Macht zu tun. Reich gewordene Nomaden kaufen sich Häuser. Solche, die verarmen, werden Diener von Sesshaften. Der europäische Agnatisierungsprozess (Duby, 1985) geht einher mit der zunehmenden Sesshaftigkeit der ehemals reisenden Feudalherren. Gleichzeitig wird die Frau abhängiger. Sie wird der Kontrolle des zunehmend territorial definierten herrschenden «Hauses» und der männlich definierten «Abstammungsideologie» unterworfen. Die Frau dient der neuen «sesshaften», männlich definierten Macht als Gattin und Mutter. Die Gewalt der Agnatisierung macht die Frau «dingfest». Wandernde Frauen werden zu Ausnahmen erklärt und gesellschaftlich abgewertet (zur Immobilisierung von Frauen siehe auch Meillassoux, 1975).

Migration ist kein neues Phänomen. Und doch staunen wir, wenn sich unter den neolithischen Funden (3900-3500 v. Chr.) in der Ostschweiz ein Dolch, bestehend aus Rohmaterial von der Adriaküste, findet. Projizieren wir nicht fast unbemerkt ein Bild von Sesshaftigkeit in die Jungsteinzeit? Obwohl wir ziemlich sicher wissen, dass auch Ötzi unterwegs war, denken wir nicht spontan, dass damals Handelsbeziehungen und Reisen über weite Strecken, in halb Europa stattgefunden haben. Es scheint, wir unterschieben unseren Vorfahren Sesshaftigkeit und Ortsgebundenheit. Was neu ist, ist nicht das Phänomen der Mobilität an sich, sondern die grosse Zahl. Die unreflektierte Projektion westeuropäischer Vorstellungen von politisch und ökonomisch begrenzten Räumen - kulminierend in der Nation - hat auch die Annahmebildung der ethnographischen Forschung beeinflusst. Man erwartete, begrenzten Ethnien mit Namen und territorialer Verbundenheit zu begegnen. Marshall Sahlins hat uns die Augen dafür geöffnet, wie die Ethnologie geneigt war, nomadisierende Jäger- und Sammlergesellschaften, 
die aus Gründen der Mobilität einen sehr saloppen Umgang mit Besitz hatten, mit Armut und Primitivität gleichzusetzen. Im Hintergrund dieser Einschätzung genau genommen muss man von Abschätzung reden - wirkten die Bilder des durch die Industrialisierung hervorgebrachten westeuropäischen Lumpenproletariats. Die Jäger und Sammler wurden zu Empfängern von unwillkommenen Gefühlen dem eigenen Lumpenproletariat gegenüber.

Verbundenheit mit der Scholle und Aufbau von Eigentum gehören zum Bild von Peasant-Gesellschaften. Den Bauern obliegt die Organisation der Erhaltung. Sie verknüpfen den Ertrag mit der Lagerung über die Zeit hinweg. Die Lagerung wiederum ist ortsgebunden. Wenn wir aus dieser Peasant-Perspektive theoretisieren, so ist uns jede Migration ein Greuel. Sie stört die Organisation der Festlegung von sozialer Identität. Paradoxerweise scheint der Begriff der «Identität»-als etwas mit sich Gleichbleibendes für gesellschaftliche Kontrollsysteme - in einer migrantischen Welt zunehmend bedeutender zu werden. Und so reimen sich Festschreibung und Substanzvorstellungen mit Kontrolle besser als prozessuale Dynamiken. Wenn soziale Zuschreibungen statisch und nicht in Kräften gedacht werden, laufen wir Gefahr, die lebendigen Aspekte der Migrationsdiskussion zu verpassen. Ich möchte die dynamischen Beziehungen betrachten, welche Menschen mit sich selbst und mit ihren Umgebungen eingehen.

Wie schwierig es sein kann, diesem Ansatz Geltung zu geben, zeigt sich unter anderem darin, dass wir unseren Vorfahren statische Weltbilder unterstellen, obwohl viele von uns in der eigenen Familie Migranten haben. Mir scheint, in der Schweiz wird die Familienvergangenheit in einem Peasant-Modell konstruiert, und die starken Wanderungsbewegungen der Bevölkerung der Bergkantone bis zum Beginn des 20. Jahrhunderts kommen kaum vor. In wie vielen Walliser und Engadiner Familien mussten über Jahrhunderte hinweg Arbeitskräfte emigrieren. Wie viele Bergbauern waren eigentliche Transhumante, die jedes Jahr im Sommer mit den Tieren ihren Wohnsitz auf die Alp verlegten?

Wir kennen die mächtige Metapher «Haus», die in unserer Geschichte eng mit dem Leib und seiner Befindlichkeit in der Welt verwoben wurde. Die Sesshaftigkeit, das «Haus», repräsentiert das «Da». Migration hingegen bedeutet die Identifikation mit dem «Fort». Theoretisch können wir annehmen, dass der Verlust und das Wahrnehmen Ich bin an einem anderen Ort, die Chance, zum Denken zu kommen, befördert. Dies ist möglich, wenn das verlorene «Da» erlebt und erkannt, unter Umständen sogar betrauert werden kann. Es kommt der Topos eines «armen Mannes vom Toggenburg» und eines Felix Platter in den Sinn, mit 
ihrer Klage, von der Brust der Mutter getrennt, in die Ferne getrieben, müsse man mit der Milch aus dem Kuhhörnlein vorlieb nehmen.

Wenn wir unter einem Zuviel an Mobilität leiden, so hat es mit dem Affekt des Verlustes eines gewohnten Standpunktes, dem Verlassen vom «Haus» zu tun. Die Globalisierung berührt diesen Pol unseres Erlebens oft stärker, als dass sie die Zuversicht über die Möglichkeiten verschiedener Hinblicke auf erweiterte und dynamische Verbindungen geöffnet hätte. Offenbar fällt es uns schwer, die Verbindung zwischen psychischen Inhalten zu konzeptualisieren, wenn wir uns gezwungen fühlen, die Umgebung, die kulturelle und gesellschaftliche Realität als instabil, mobil, nicht in Konstanten, die an einem fixierten Standpunkt festzumachen sind, hinzunehmen, und dies alles während wir uns selbst als einen Teil der Dynamik erkennen müssen.

Meine Überlegungen beziehen sich im Wesentlichen auf nichtlineare Bewegungen des Primärprozesses und auf das Lustprinzip. Je mehr wir uns vor primärprozesshaften Vorgängen fürchten, je weniger wir uns ihnen hingeben wollen, desto mehr ängstigen uns Bewegungen, die in der globalisierten Dynamik und Topologie schwer fassbar scheinen. Der Cyberspace scheint dem Traum näher zu stehen als dem Realitätsprinzip.

Die Ideologie der Sesshaftigkeit, mit ihrem blinden Fleck der Gewalt, die darin gefangen ist, erschwert bis verunmöglicht das klare Erkennen der Möglichkeiten und Verwirrungen in der mobilen Erweiterung. Die neolithische Wendung zur Sedentarisierung hat soziale Macht domestiziert. Wird die konsequent bearbeitete Erde zum Produktionsmittel (Meillassoux, 1975) und die Lagerhaltung der Produktion zentral, so wird das «Haus» zum «Kontrollort» für Güter und zum Schutz für Reichtum und Besitz. Heute, denke ich, ist das «Haus» in diesem Sinne obsolet, obwohl noch so getan wird, als hätte es die Bedeutung der Peasants. - Ein anderer Aspekt der Gewalt ist die Spaltung von «sesshafter Häuslichkeit» und frenetischer Mobilität. Nur eine Verleugnung der zahllosen Abhängigkeiten von Verbindungen in der Luft, auf und unter der Erde (Telefonie, Elektrizität, Strassen) kann zur Illusion von häuslicher Autarkie führen. Was in der Ideologie der Sesshaftigkeit, dem «Haus», dem Bollwerk Nation und Rasse kulminiert, sind die Konzentrationslager (vgl. auch Agamben, 1995).

Die Geschichte der Psychoanalyse ist mit dem Thema Migration verknüpft. Sowohl was die persönlichen Migrationsgeschichten Einzelner anbelangt, wie beispielsweise von Freud, der wie viele andere von der Peripherie des alten ÖsterreichUngarn in die Hauptstadt gezogen ist, seine soziale Aufwärtsmobilität im Streben nach bürgerlicher Sicherheit, wie auch durch die Emigration der Psychoanalytiker 
im Nationalsozialismus. Alle Zwangsemigrierten mussten - ob in Nordamerika, Südamerika, Afrika oder China - mit der anderen Sprache, der Unsicherheit und Angst leben. Obwohl man annehmen muss, dass ihr Theoretisieren davon nicht unbeeinflusst geblieben sein kann, stellen wir fest, dass eine vor allem an der zweiten Topik orientierte Ausrichtung ausgebaut wurde. Die erzwungene Emigrationserfahrung und die Existenzbedingungen im Übergangsraum waren zu katastrophal. Der lineare Entwicklungsgedanke, als eine progressive Differenzierung, trägt die Möglichkeit des Zurück in sich, des Rückfalls und der Pathologie. Und so gelangen wir zum Konzept der Regression. Regression kann für den Flüchtling das Inferno und der Tod heissen. Das psychoanalytische Konzept lädt sich mit einer Erfahrung in der Wirklichkeit auf und verleiht ihm eine existentielle Relevanz. Das «Vorwärts» und «Fort» verbindet sich mit dem Überleben, das «Rückwärts» mit dem Tod. Es kann keine Dialektik von «Da» und «Fort» geben.

Was Bion mit der Oszillation von $\mathrm{PS} \Leftrightarrow \mathrm{D}$ einführt, ist ein dialektischer Umgang mit dem Hin und Her, Vorwärts und Rückwärts. Bion theoretisiert Brüche wie Caesura, katastrophische Veränderungen und Fragmentierungen. Es ist zu vermuten, dass diese theoretischen Anliegen mit seinen Lebensbrüchen zu tun haben (vgl. Torres \& Hinshelwood, 2013). Einschneidend erfuhr er den ersten Weltkrieg als Panzeroffizier und sagte später, dass er «damals starb». Als kleiner Bub lebte er um die Jahrhundertwende in Indien. Sein Vater war Engländer mit Deutschschweizer Wurzeln, seine Mutter Angloinderin. Die Gruppen, mit denen er in Kontakt kam, waren hochkastige Inder und nicht minder rigide geformte Gruppierungen bei den Engländern. Sein Vater nahm als Ingenieur die ganze Familie auf seine Baustellen mit, die weit weg von der Stadt waren. Dort bewegte sich der kleine Bub, der Hindi und Englisch sprach, mit Leichtigkeit von Gruppe zu Gruppe. Als Achtjähriger wurde er von der Familie und Indien getrennt und kam ins Internat nach England (Bion Talamo, 2011).

Bion ist - denke ich - ein «moderner» Theoretiker, weil seine Herangehensweise unserem Bedürfnis entspricht, den Wandel, das «Werden» und die damit verbundenen Denkmöglichkeiten in einer als «instabil» erkannten Umwelt, die paradoxerweise kontinuierlich für Brüche sorgt, zu untersuchen. Nach Bion impliziert das Denken ein Alternieren von Momenten der Desintegration und der Integration; er verweist auf diesen Wechsel mit den Zeichen PS $\Leftrightarrow$ D. Jeder Augenblick der Sicherheit (D) wird gefolgt von einem Bruch (PS), der die Fähigkeit erfordert, eine Situation der Unsicherheit und mangelnden Stabilität zu ertragen, «in einem Spiel der Reprisen, welches das Werden gegenüber dem Sein betont, das Denken gegenüber dem bereits Gedachten» (Neri, 2006, S. 159). Für Bion liegt die Wahrheit 
im kontinuierlichen Werden (1984). PS $\Leftrightarrow \mathrm{D}$ modelliert die Oszillation zwischen der Notwendigkeit, dem Informen Form zu geben, und dessen Wiederauflösung in Kauf zu nehmen. Im Gegensatz zu Melanie Klein, der zufolge sich PS und D auf eine Entwicklungsstufe bezieht, bezeichnet Bion damit eine seelische Funktionsweise, nahe den psychotischen Fantasien und Mechanismen, die er in Gruppen beobachtet hat. Er vergleicht die instabile und verwirrte Gruppe mit einer Psyche, die versucht, die einzelnen Teile in eine erträgliche Beziehung zueinander zu setzen. Die «stabile» Gruppe hingegen gleicht mehr dem, was Freud unter den neurotischen Mechanismen versteht. Mit den Gedanken zur «Mobilität» betrachte ich einen Bereich des psychischen Funktionierens, der am Scharnier jeglicher psychischer Veränderungen beteiligt ist. Ich bewege mich also nicht entlang einer diachronen Achse. Diesen Ansatz wählt Freud in Totem und Tabu, wenn er für die Gruppe eine Art von Kindheit mittels der Urhorde annimmt (Freud, 1974). Die kannibalische Tötung des Vaters bedeutet dann die Primärszene jeder weiteren psychischen Entwicklung (vgl. Neri, 1985). Bion knüpft ebenfalls an Totem und Tabu an und findet neben dem Familienmodell, das für Freud das Grundschema der Gruppe darstellt, eine andere Struktur, die er protomental nennt. Es ist der Punkt, wo Freud in "Massenpsychologie und Ich-Analyse» sagt, dass sich die «individuelle Abgrenzung und Eigenart» (1974, S. 79, 81) verliert.

An dieser Stelle möchte ich kurz etwas zum Begriff «psychotisch» einfügen. Ich ziehe dem Begriff «psychotischer Kern», den des «psychotischen Funktionierens» vor. Das heisst, ich betrachte nicht einen verdrängten Teil, sondern einen Prozess, der entlang der Kontaktbarriere permanent stattfindet. Auch mit dem Modell container $\Leftrightarrow$ contained gefasst, ist die Unterscheidungslinie zwischen Verwerfung und Spaltung auf der einen Seite, und Verbindung und Integration auf der anderen Seite, das Resultat eines kontinuierlichen Prozesses, worauf ich hier jedoch nicht weiter eingehen will. $\mathrm{P}-\mathrm{S} \Leftrightarrow \mathrm{D}$ bezieht sich ebenfalls auf die Kontaktbarriere und ihre flexible Funktion im Trennen und Vereinen von verschiedenen Elementen. P-S trennt und differenziert, und D verbindet in «improvisierender und kombinatorischer Hexerei» (Grotstein, 2007, S. 51). Grundsätzlich ist P-S $\Leftrightarrow \mathrm{D}$ die Notation des «Lernens aus Erfahrung» - die Emotion.

Nach Bion ändern gewisse Grundfunktionen mit dem psychischen Wachstum nicht. Einer dieser Aspekte des psychischen Lebens, der in diese Kategorie fällt, ist die Angst vor der äusseren Realität - und der physischen inneren Realität -, die durch die Sinne wahrgenommen wird, die das Gesamt der Grundinformationen konstituieren, auf die wir zum Überleben zurückgreifen (Bion Talamo, 2011, S. 238). Angesichts der Schwierigkeit, die Auswirkungen der 
globalisierten Netze auf den Menschen zu repräsentieren, geht es meiner Meinung nach erst recht darum, uns auf unsere Erfahrungen zu besinnen. Als Analytiker sind wir täglich mit dem Nicht-Repräsentierbaren konfrontiert. Es scheint mir deshalb sinnvoll, nach Konzepten Ausschau zu halten, die uns erlauben, «sesshafte Klumpen», nucleo aglutinado (Bleger, 1967), Bollwerke (Baranger \& Baranger, 1960-1961; vgl. Bazzi, 2012), die «primitive Struktur» oder eine «undifferenzierte Matrix» in Bewegung zu bringen. Geht es uns denn nicht um die «Mobilität» von Gefühlen?

Mir ist ein über hundert Jahre alter Text wieder in den Sinn gekommen, an dem ich meine Überlegungen zeigen möchte. Marcel Mauss, der «Vater der französischen Ethnologie», hat im «Essay sur les variations saisonnières des sociétés eskimo» (1906) mit der teilweisen Mitarbeit von Henri Beuchat eine nomadisierende Lebensweise theoretisiert, und die von ihm gefundene soziale Organisation als duale Morphologie bezeichnet, die meiner Meinung nach noch heute von Bedeutung ist. Ein Architektursoziologe meint sogar, sie sei von brennender Aktualität: «[...] l'apport de Mauss tout à fait important, novateur pour ne pas dire prémonitoire, à la mise en évidence des faits de mobilité liant la demeure à ses territoires. Saisis dans leurs dimensions temporelles et sociales, ils traversent une problématique d'une brûlante actualité [...]» (Pinson, 2012). Der Essay von Mauss hat mich dazu inspiriert, auf das, was ich «Ideologie der Sesshaftigkeit» nenne, einen anderen Blick zu werfen. Aus Platzgründen und um mich auf meine Argumentationslinie konzentrieren zu können, verzichte ich auf eine weitere wissenschaftshistorische und kritische Einordnung des Essays. Ich werde das ethnografische Material in einerWeise brauchen, die dem hier beschriebenen psychoanalytischen Denken mehr verpflichtet ist als einer positivistischen Herangehensweise. Es geht mir um eine mögliche Darstellbarkeit der Mobilität, sei sie ethnologisch gefasst als Nomadismus, als Migration, sei sie psychoanalytisch beschrieben als Oszillation zwischen Strukturierung und Entstrukturierung.

Mauss findet die Inuitgesellschaften bemerkenswert, weil sie jahreszeitlich in ihrer Behausung und der Art der Niederlassung einen völligen Wandel durchmachen und sich verschieden gruppieren. Die Zusammenschau der abwechselnden und völlig unterschiedlichen Lebensweisen der Inuit, die im Sommer in Kleinfamilien riesige Gebiete durchwandern und im Winter in grossen Gruppierungen sesshaft bleiben, halte ich für einen genialen Ansatz. Im Vorwort zum Werk von Mauss bezeichnet Claude Lévi-Strauss dessen Definition des sozialen Lebens als «Welt symbolischer Bedeutungen». Mauss verbindet Ökologie mit Religion, mit Ritualen und kollektiven Zeremonien, und zeigt die Interdependenz von so verschiedenen 
«Tatsachen» wie Sozialstruktur und kollektiven Repräsentanzen. Er stellt fest, dass die ihm vorliegenden Arbeiten zu den Inuit entweder geografisch und auf die ausschliessliche Wirksamkeit der Beziehung zum Boden fokussiert sind, oder aber nur die Wanderungen beachten. Karten sind z.T. unbrauchbar, da sie Siedlungen suggerieren, wo es sich jedoch um Wanderungen handelt. Mauss' Analyse zeigt, dass der Stamm keine territoriale Einheit bildet. Die Stammesaggregate haben «die meiste Zeit sehr unscharfe und unbeständige Gestalt», «man weiss nicht, wo sie beginnen und wo sie enden; sie scheinen sich sehr leicht miteinander zu vermischen und sich miteinander proteushaft zu kombinieren» (1978, S. 198). Schwierig wird es, wenn festgestellt werden soll, wer wen als Fremde bezeichnet, da dies mit Grenzen in Verbindung gebracht werden muss. «Die Zusammensetzung der Eskimogesellschaft hat von ihr selbst her etwas Unpräzises und Schwankendes, und es ist nicht leicht zu unterscheiden, aus welchen abgeschlossenen Einheiten sie gebildet wird» (a.a. O., S. 196). Dies bezieht sich auf Sprache, also linguistische Einheiten, Eigennamen von Stämmen, und territoriale Verbundenheit und Einheit von politischen Gruppen. Zu den kollektiven Namen, die sich Stämme geben, sagt Mauss: «[...] ganz offenkundig ist die Nomenklatur in diesem Punkt von einer extremen Unbestimmtheit» (a.a.O., S. 196). Und weiter: «Dieselbe Unbestimmtheit herrscht hinsichtlich der Grenzen, wo sich doch gerade in dieser Hinsicht die Einheit einer politischen Gruppe, die ein Bewusstsein ihrer selbst hat, am schärfsten bekundet» (a.a. O., S. 198). Dann findet Mauss die «wirkliche territoriale Einheit» in der Siedlung. «Es hat sogar den Anschein, als gebe es eine Art regelmässiger Rückkehr des Greises zu seinem Geburtsort» (a. a. O., S. 199). An diesen Zitaten wird deutlich, wie stark die damalige Ethnologie vom Denken in fest abgegrenzten Räumen geprägt war. Trotz seiner Kritik am deutschen Geografen F. Ratzel, der den «tellurischen Faktor» privilegiert, muss Mauss die «territoriale Einheit» klarstellen. Deshalb hält er die Durchlässigkeit und Mobilität zwischen den Siedlungen für eine Ausnahme. «Sie hat ihre Ursache immer in bedrängenden vitalen Notwendigkeiten, so dass, da jede Abweichung sich leicht erklären lässt, die Regel nicht verletzt scheint» (a.a. O., S. 199). Die detaillierte Beschreibung der Siedlungen zeigt deren hohe Fluktuation. Starke Auswanderungen und dann wieder starke Zuwanderungen, führen zu einer schwankenden Bevölkerung (ich benütze das ethnografische Präsens). Wegen den weiten Wanderungen - die Inuit leben von der Jagd oder dem Fischfang, sie brauchen offenes Meer oder vereistes Land - können die Siedlungen nur klein bleiben, stellt Mauss fest.

Im Sommer bewohnt eine Familie ein Zelt. Im Winter wohnen bis zu zehn Familien im Langhaus. Beim Langhaus führt ein unterirdischen Gang in einen 
grossen Raum, der ein bis zwei Bänke aufweist und in Abteile unterteilt ist, in denen die einzelnen Kleinfamilien leben. Ebenso strukturiert ist der Iglu. Zwei, drei Iglus werden mit einem Gang verbunden, dessen Öffnung halb unterirdisch ist. Dann gibt es im Winter noch ein Gemeinschaftshaus, «kashim» genannt, wo sich alle Bewohner der verschiedenen Langhäuser in einem Raum treffen können. Die Winterstation besteht aus mehreren nahe beieinander stehenden Häusern. In einer Fussnote erwähnt Mauss, dass es überall noch Mischformen von Zelt und Haus gibt "die für bestimmte Zwecke und vorübergehend in Gebrauch sind» (a. a. O., S. 227). «Fast überall kommt es vor, dass man auf diese Mischkonstruktionen zurückgreift, und zwar namentlich dann, wenn man während eines Standortwechsels sogar im Sommer durch eine Reihe von schlechten Tagen genötigt ist, einen Unterstand zu bauen» (a. a. O., S. 228, kursiv D. B.). Im Winter sind die Behausungen dicht beieinander, ja als «hätten sie ehedem aus einer Art grossem und zugleich vielgliedrigem, einzelnen Haus gebildet werden können» (a. a. O., S. 231). Im Sommer vollzieht sich eine Vereinzelung der Familien, die sich weit voneinander entfernen und beachtlicheWanderungen unternehmen. Mauss bemerkt, dass die Sommerlager und die Wanderungen, weil vorübergehend und unbeständig, eine extreme Mobilität der Familien und Individuen verlangen. Dann ist der Bewegungsraum entlang den Küsten und im Landesinneren entlang den Flüssen und an Binnenseen für die Jagd fast unbegrenzt, im Winter hingegen höchst eingeschränkt. «Die Bewegung, welche die Gesellschaft belebt, ist synchron mit der des umgebenden Lebens» (a.a.O., S. 240). Abgesehen von den Karibus leben die Inuit vor allem von den Meerestieren, hauptsächlich von Walfischen. Sie brauchen im Winter und Frühjahr offenes Wasser oder vereistes Land für die Robbenjagd. Oder es wird bei einem Loch im Eis, wo die Robbe Atem holt, mit einer Harpune gejagt. Im Sommer sind Jagd- und Fischgründe im Süsswasser. Alle diese Bedingungen sind unbeständig und unsicher.

Im Sommer gibt es keine Religion. «Alle Mythen, die [...] im Winter das Bewusstsein der Eskimos erfüllen, scheinen während des Sommers vergessen. Das Leben ist gleichsam säkularisiert» (a. a. O., S. 243). Dagegen «lebt dieWintersiedlung sozusagen in einem Zustand fortwährender religiöser Überspanntheit» (ebd.). "Alles in allem kann man sich das ganze Leben im Winter als eine Art grosses Fest vorstellen» (a. a. O., S. 243-44). Mauss benützt das Wort «Paroxysmus» - ein Bild, das die Folge von sich steigernden Ausbrüchen eines Vulkans evoziert -, um zu sagen, dass das geringste Ereignis, aber auch jedes kollektive Unglück, wie ein Sturm, Flucht der Jagdtiere, Bruch des Eises etc. die Schamanen zum Eingreifen nötigt. Das religiöse Leben ist nicht nur intensiv und kollektiv, sondern die Feste, 
die im kashim stattfinden sind ganz Sache der Gruppe: «Es ist die Gruppe, die sich in ihnen ausdrückt» (ebd.). Beim Blasenfest wirft man «alle Blasen aller Meerestiere, die von der gesamten Gruppe während des ganzen Jahres getötet wurden, ins Meer» (a.a. O., S. 245, kursiv M. M.). Man nimmt an, dass die Tierseelen in den Weibchen wiedergeboren werden. Anlässlich eines Festes der Toten bittet man die Toten, sich in den Trägern desselben Namens zu reinkarnieren. Neugeborene erhalten Namen von eben Verstorbenen. Dann überhäuft man die gleichnamigen Lebenden mit Geschenken und verabschiedet die Seelen, die ins Land der Toten zurückkehren. Mauss will die Verbindung und Kontinuität der Generationen mit der Subsistenzwirtschaft aufzeigen. Diese Verbindungen sind auf dem Hintergrund der Trennungen, die Inuitfamilien im Verlauf des Jahres durchmachen, besonders bemerkenswert. Durch die Zerstreuung und Vereinzelung im Sommer und das intensive Kollektivleben im Winter verändern sich die sozialen Zugehörigkeiten im jahreszeitlichen Rhythmus und man kann annehmen, dass das Hin und Her der ändernden Zugehörigkeiten mit psychischen Prozessen einhergeht.

Im Verlauf eines der kollektiven und öffentlichen Feste der Wintersonnenwende werden alle Lampen im selben Augenblick gelöscht und wieder angezündet (kursiv M. M.). Dazu muss man wissen, dass jede Familie nur eine Tranlampe hat, die im Besitz der Frauen ist. Im Sommer gibt es in jedem Zelt eine Lampe und im Winter entsprechend mehrere Lampen im Langhaus. Zentrieren wir für einen Moment unsere Überlegungen nicht um die ändernde Behausungsform, sondern betrachten wir das bewegliche Gut «Lampe» als wäre es ein «selected fact», so erhält das Element des kollektiven Löschens eine besondere Dynamik. In Cogitations schreibt Bion, $\mathrm{P}-\mathrm{S} \Leftrightarrow \mathrm{D}$ sei die Suche nach dem signifikanten vereinigenden Fakt, der «Ursache» genannt wird, wenn die Zeit eine Komponente ist (1994, S. 104). Dazu fällt mir Bions Verwendung einer Briefstelle von Freud an Lou Andreas Salomé ein. Es geht um das Verdunkeln, um besser sehen zu können. "Freud said that he had to 'blind myself artificially to focus all the light on one dark spot'. This provides a useful formulation for describing the area I wish to cover by F. By rendering oneself 'artificially blind' through the exclusion of memory and desire, one achieves F; the piercing shaft of darkness can be directed on the dark features of the analytic situation" (1984, S. 57). Mit F bezeichnet Bion «Faith». Grotstein sagt, Faith entsteht da, wo das Kind die abwesende Brust betrauern kann (2007, S. 316). Bion nimmt an, dass das Kind in die Positionen geboren wird, das heisst simultan in D wie auch P-S. "For convenience I propose to call this state, which is neither the paranoid-schizoid position nor yet the depressive position but something of each, the Positions. I shall further suggest that the process of 
discovery or selection of the harmonizing fact or, as I should prefer to regard it, its ideational counterpart, cannot be initiated or maintained without the mobilization of the mental process of dreaming" (1994, S. 215). Ich verstehe das so, dass wir mit Aussicht auf den Objektverlust geboren werden (vgl. Grotstein, 2007, S. 310). Bion arbeitet hier an der Freud'schen «gleichschwebenden Aufmerksamkeit». Er fügt hinzu, dass es dann, wenn etwas besonders im Dunkeln liegt, nicht darum geht, sich am Bekannten und an Sinneswahrnehmungen zu orientieren. So gesehen könnte diese Methode - so Bion - zum Missverständnis verleiten, es gehe um eine völlige Verleugnung der Realität. Jedoch suche der Analytiker nach etwas anderem, als dem, was normalerweise unter Realität verstanden wird (a.a. O., S. 43).

Wenn die Inuit in der Gruppe die Lampen gelöscht haben, wenden sie sich diesem anderen zu. Mauss stellt fest, dass "Alle Feste [...] von auffälligen Erscheinungen sexueller Freizügigkeit begleitet [sind]» und dass es die orgiastische Gemeinschaft «vielleicht die innigste, die es gibt» sei, wo es «zu einer Art Verschmelzung der individuellen Personen ineinander [kommt]» (Mauss, 1978, S. 246). Es ist bemerkenswert, dass Mauss diese Zustände als soziologische Tatsache beschreibt, es ist für ihn eine «Gemeinschaft». Dass regulierte und symbolisch überformte Elemente in diesen Ritualen vorkommen, halte ich für selbstverständlich. So gibt es Berichte, dass der Frauentausch entsprechend den Namen, die auf Mythen bezogen sind, vollzogen wurde. Das heisst, Männer und Frauen wurden so vereint, wie die mythischen Ahnen, deren Namen sie gegenwärtig tragen und deren Repräsentanten sie sind (a.a.O., S. 260). Für Mauss stellt der sexuelle Kommunismus die Entfernung von derVereinzelung im Sommer dar. Als Einschub möchte ich zu bedenken geben, dass die exotisierende, und damit distanzierende, Kenntnisnahme vom Brauch des «wife swapping» der Inuit möglicherweise dem Bias zu verdanken ist, der durch die Brille der Sesshaftigkeit entsteht. Vergleicht man das Geschehen mit dem Aufbau der Mozart-/Da Ponte-Oper «Così fan tutte», lernt man, dass sich das wife swapping nur mittels des Tricks der Abreise bewerkstelligen lässt. Wir können annehmen, dass auch die Einbindung in Triebziel und Triebobjekt einer drohenden Formlosigkeit der Erotik und Libido bedarf, um ausgedrückt und erzählt werden zu können. Mozart hat, im Vergleich zu den ethnographischen Berichten, die wir zu den Bräuchen der Inuit erhalten haben, den umgekehrten Weg gewählt. Doch zurück zum Lampenritual. In der Dunkelheit stellt sich die Frage, wie Kreativität, wie Schöpfung möglich ist. Dies ist ein anderer Ansatz als unsere Art von Empirie. Dieses Denken, das sich engstens mit dem Ausdruck verwebt, bedingt eine momentane Entledigung des Erfahrungswissens. Man kommt zu einer anderen Ebene des Nicht-Wissens, der Frage, warum bleibt 
das Jagdglück aus, warum Verschwinden die Tiere, man gibt sich P-S $\Leftrightarrow \mathrm{D}$ hin. Die kollektiven Feste sind so gesehen auch Ausdruck dessen, was man nicht weiss. "To what extent is the constant transition from paranoid-schizoid to depressive position and back again the essential mechanism of learning throughout life?" (Bion, 1994, S. 185).

In diesem Zusammenhang ist es nicht unwichtig, sich daran zu erinnern, dass alle Schamanen reisen. Sie reisen zum Mond, zum Haus der Winde, sie tauchen ins Meer ab. Margaret Lantis stellt zur Frage, ob der Schamane schizophren sei - die dazumal unter Ethnologen diskutiert wurde -, fest, wenn er es ist, so ist er es jedenfalls kontrolliert, und er erholt sich. Er wird nicht unwiderruflich desorganisiert oder so paranoid, dass seine Mitmenschen ihn nicht mehr aushalten können. Die Gemeinschaft akzeptiert die Möglichkeit eines dualen Funktionierens, das heisst, in Beziehung zur sozialen Umwelt und zur natürlich-übernatürlichen Umwelt (Lantis, 1950). Ich möchte hier das Augenmerk auf die Bewegung richten, auf die kulturelle Akzeptanz des Oszillierens zwischen einem desorganisierten Zustand und einem Zustand der Rückkehr zum «wieder Anzünden der Lampen». Lantis spricht in dem Zusammenhang von rationalem Lernen und Fluidität. “To them, their view of life is a series of rational conclusions based on observation and experience. It can be modified, added to by new experience, hence is relative" (a. a. O., S. 319). Dann gibt es aber die Unsicherheiten "[...] beliefs regarding the world perhaps are symbolizations of such uncertainties” (ebd.). Lantis meint, die Religion sei «stark und befriedigend» gewesen, "to last through long migrations and subsequent isolation [...] symbolism of fluidity, changeability of life, even of unreliability, and a lack of symbolism indicating attachment to the land are noticeble. [...] This fluidity probably reflects the ancient as well as modern mobility of the Eskimos, who must be continually moving about in search of food and who most often seek that food from sea" (a. a. O., S. 334-335).

Der Gegensatz des Winter- und Sommerlebens wirkt auf die Mentalität der Gruppe. Bei Festen haben sich die Leute in zwei Lager aufgeteilt. Zu den einen gehören diejenigen, welche im Sommer geboren sind, zu den anderen jene im Winter geborenen. Die letzteren heissen Schneehühner, die Sommergeborenen sind die Eiderenten. Auch in den Mythen werden die Tiere in Sommer- und Wintergruppen aufgeteilt. Sommer- und Winterdinge werden so stark getrennt, dass jede Art von Mischung zwischen ihnen verboten ist, wie beispielsweise die Berührung zwischen dem Fell des Karibu und dem Seehundfell. «Wenn der Sommer beginnt, darf man kein Karibu (ein Sommertier) essen, ohne dass man vorher alle im Winter getragenen Kleider abgelegt und neue Kleider oder zumindest solche 
angezogen hat, die während der Jahreszeit der Jagd auf die Robben nicht berührt worden sind» (Mauss, 1978, S. 248).

Ich möchte diese Trennungen und Tabus in relationaler Weise verstehen. Sie konzeptualisieren die Ablösung, die Tatsache, dass immer wieder das Eine und das Andere verschwindet. Im Verbot macht man die Bewegung auf einer symbolischen Ebene mit. Was einmal genossen werden kann, verschwindet wieder und wird unerreichbar. Die Zuordnung von Winter- und Sommertieren, die strikte Trennung ermöglicht eine bezugnehmende Sinngebung. Dass die Inuit genau zwischen Winter- und Sommersymbolen trennen, verweist auf eine grundsätzliche Hybridität oder Mobilität der «Inhalte», die zur kulturellen Differenzierung taugen. Es kommt mir die Kritik von Winnicott an Melanie Klein in den Sinn, sie sei mehr an der Bedeutung des Spiels der Kinder als am Akt des Spielens selbst interessiert gewesen (Grotstein, 2007, S. 267; vgl. auch Ferro, 2013).

Bezeichnenderweise sind die einzigen Riten des Sommers Geburts- und Todesriten. Das heisst, die zentralen Erlebnisse von Ankunft und Abschied werden symbolisch geformt. Wenn man so will sind es Rituale, welche die Unsicherheit der mobilen Strecke fassen. Die Sommerfamilie wird als sehr prekär, was ihre gegenseitige, auch technische Abhängigkeit betrifft, geschildert. Wenn einer der Partner stirbt, ist kaum ein Überleben möglich. Die Inuit lebten fortwährend mit dem Tod (vgl. Briggs, 1970).

Das Bild, das Mauss von den Inuitgesellschaften zeichnet, macht deutlich, dass sich Mobilität nicht nur auf die Wanderungen im Sommer bezieht, sondern auf die Rhythmizität und die «Beweglichkeit» sozialer Formen der Gesellschaft überhaupt. So ist die Sommerfamilie patriarchalisch organisiert, währenddessen die Wintergemeinschaft, die Housemates des Langhauses, egalitär leben. Im Abwechseln der sozialen Formen geht es also um eine Mobilität innerhalb derselben Gesellschaft.

«Intensität» ist eines der wiederkehrenden Worte von Mauss für die Wintergesellschaft. Die Menschen leben in fortdauerndem Austausch und verausgaben sich. Mauss führt das Beispiel einer Erzählung an, wo eine Frau froh ist, dass sie nach dem Winter wieder allein sein kann. Im Sommer leben, fischen und jagen sie «individualistisch» und pragmatisch. Im Winter ergreift die Gruppe die Möglichkeit, den Wirkungen der vereinzelt erlebten Unwägbarkeiten kollektiv Ausdruck zu geben. Im winterlichen kollektiven Festleben werden in der Gruppe die Spuren der Trennung und der Angst «geträumt». Im riesigen «Sommerfeld» gibt es seltene Begegnungen der einzelnen Menschen, sodass das Leben sich in der «technischen» Abwicklung erschöpft. Das «Winterfeld» ist sehr klein und konzentriert und äusserst 
dynamisch. Die Beobachter empfinden das Leben der Inuit dann als Ausschweifung und Überspanntheit. Könnte man das berühmt gewordene Paar «Verausgabung und Wiederherstellung» von Mauss mit $\mathrm{P}-\mathrm{S} \Leftrightarrow \mathrm{D}$ parallelisieren? Dies stellt sich hier als Nebenfrage, der nachzugehen den Umfang der Arbeit sprengen würde.

Das Mauss'sche Modell der jahreszeitlichen Mobilität der Inuit regt an, darüber nachzudenken, wie Menschen mit wechselnden Standorten und Strukturen umgehen. Zum Dimorphismus oder dem auch bei Mauss nicht ganz durchgehenden binären Bild und dem dualen Modus habe ich den relationalen Gesichtspunkt herangezogen. Die relationale Interdependenz findet sich auch im Mythos der Sedna (Meeresgöttin der Inuit) im Bezug auf die Tiere, die gejagt und gefischt werden. Aus den abgehackten Gliedmassen von Sedna, der Mutter der Meeresgeschöpfe, entstanden alle Meerestiere. Ich halte es für eine ethnozentristische Interpretation, wenn dafürgehalten wird, dass die Jagd genau das sei, was die Inuit brauchen; weil sie aber den vielen Gefahren und Zufällen derart ausgeliefert sind, können sie nicht anders, als sie mit religiösen Mitteln zu «bannen». So wird ein Grundunterschied suggeriert, der die Angst in eine «primitive» Produktionsweise und prekäre Ökologie verweist, wo Unsicherheit regiert, währenddessen wir eine rationale Sicherheit herstellen können. In der Séance reist der Schamane zu Sedna und beruhigt sie. Diese Bewegung ist keineswegs ein Bannen von Angst. Ich finde den Essay von Mauss so inspirierend, weil ich der Ansicht bin, dass die primärprozesshafte Anverwandlung und Bearbeitung sozialer und technischer Anforderungen genauso wichtig ist, wie eine kognitive und rationale Verarbeitung. Mit «technisch» meine ich z. B. die Atemloch-Harpunen-Jagd auf Robben oder das gemeinsame Rudern im «Umiak», dem grossen Boot, das den Frauen gehört. Es geht mir um die «mobilen» Aspekte und Funktionsweisen der Psyche, die in Wechselwirkung mit der immer stärker als «mobil» erlebten sozialen und technischen Umwelt in den Fokus geraten; die ich im Sinne der Oszillation von P-S $\Leftrightarrow \mathrm{D}$ gut gefasst finde und die ich auch beim Arbeiten am vorliegenden Text erlebe, wenn ich den mir altbekannten Essay von Mauss dekonstruiere. Was ist dekonstruieren anderes, als $\mathrm{P}-\mathrm{S} \Leftrightarrow \mathrm{D}$ ? Oder wie Claudio Neri formuliert «Ein origineller Gedanke kann mit der Zeit die Form einer Abwehr einnehmen, Emotionen können sich in konventionelle und formelle Ausdrucksformen verwandeln. Das Oszillieren $\mathrm{D} \Leftrightarrow \mathrm{PS}$ gestattet es, erneut mit den authentischen Gedanken und Gefühlen in Kontakt zu treten» (2006, S. 160).

Mauss, der immer wieder darauf hinweist, dass die geografische Lage nur eine der Bedingungen für die Zusammenziehung und Verstreuung der Inuitgesellschaften sind, stellt in den Schlussfolgerungen in Frage, ob die Jahreszeiten 
der Grund für den Rhythmus sind. Lässt man die Frage nach einem naturgegebenen Grund beiseite, so bleibt der Rhythmus Ausdruck der Bewegungen zwischen Anforderungen und Bewältigung von sozialen und technischen «Gegebenheiten». Mauss komponiert ein elegantes «Bild» von Bewegung - die Mobilität fasst er in wiederkehrenden Begriffen von binärem Rhythmus und Synchronizität. Interessant ist, dass er eine psychologische Ursache für die bewegliche Sozialstruktur heranzieht. Er meint, dass das soziale Leben auf die Individuen eine Gewalt ausübt, die sie nur eine «gewisse Zeit lang ertragen können» und die dazu führt, dass sie sich zeitweise entziehen müssen. Deshalb Zerstreuung und Zusammenziehung, Verausgabung und Wiederherstellung.

Das Schöne am Essay von Mauss ist, dass er uns eine soziale Funktionsweise aufzeigt, die auf Symbiose und Synchronizität, Egalität und Gleichgewicht ausgerichtet ist. Sein Interesse gilt nicht dem Wachstum, sondern sein holistischer Ansatz beleuchtet die Harmonie mit der Umgebung und die kollektive Aufgabe gegenüber dem individuellen Wettbewerb. Ich versuche zu zeigen, dass das Gleichgewicht in den verschiedensten Bereichen der Inuitgesellschaften kein statisches ist, sondern mit dem Ungleichgewicht oszilliert. Mauss betrachtet Symbiose und Synchronizität in Bezug auf die mobile Lebensweise, deren Rhythmus nur durch Trennungen, Abschied, Übergangszeit und Ankunft denkbar ist. Die symbiotische Mobilität ist die Verbindung mit dem saisonalen Auftauchen und Verschwinden der Tiere, die zeitgleiche Bewegung mit den Migrationsmustern der Tiere. Der saisonale Rhythmus steht in Relation zu tiefgehenden sozialen Veränderungen, wie dem Wechsel vom Leben in der Kleinfamilie im Sommer zur Gruppe im Winter. Der Mauss'sche Ansatz ermöglicht zu sagen, dass sich die Rhythmizität auf die immer wieder erneut sozial aktiv betriebene Sinngebung bezieht. Dieser Tatbestand so denke ich - ist in der Formel PS $\Leftrightarrow D \Leftrightarrow P S \Leftrightarrow D \Leftrightarrow P S \Leftrightarrow D$, die ich als fortlaufend sehen möchte, am besten dargestellt. Es geht mir um die Gleichzeitigkeit von archaischen psychischen Aspekten und strukturierten psychischen Aspekten. Ein Aspekt der «orgiastischen Winterreligion», das gemeinsame Essen, Musizieren, Tanzen und Trinken kann als protomentaler Ausdruck und «Antwort» der Gruppe auf die Trennungserlebnisse verstanden werden. Meine Annahme ist die, dass die Bewegung - vom einen zum anderen - dem Primärprozess angehört, der Oszillation zwischen Schlafen und Wachen vergleichbar, wenn man beim Erwachen nicht gleich aufspringt, sondern nochmals träumt. Wenn man wieder aufwacht, dann wieder träumt und etwas vorgeht, was man mit $\mathrm{PS} \Leftrightarrow \mathrm{D} \Leftrightarrow \mathrm{PS} \Leftrightarrow \mathrm{D} \Leftrightarrow \mathrm{PS} \Leftrightarrow \mathrm{D}$ bezeichnen könnte. 
Aus dem bisher gesagten wird deutlich, dass es mir nicht um das Verhältnis vom Individuum zur Gruppe geht (vgl. die Einleitung zu Mauss von Lévi-Strauss), sondern um eine Überlegung zu psychischen Prozessen, die theoretisch gesehen im Individuum das Gruppale betreffen und in der Gruppe die Notwendigkeit zur Formgebung und Symbolisierung. Individuum und Gruppe können nicht getrennt betrachtet werden. Obwohl sich Bion in seinen frühen Schriften für das Verhalten der Gruppe und nicht dasjenige der einzelnen Individuen interessiert, zeigen seine Arbeiten, dass der Fakt, Teil einer Gruppe zu sein, eine grundlegende Bedeutung für das Individuum und für sein psychisches Wohlergehen hat (Bion Talamo, 2011, S. 119). Unseren psychischen Apparat können wir nur mittels Filterung und Metabolisierung der eingehenden «Realität» durch einen anderen psychischen Apparat aufbauen, und wenn wir annehmen, dass es ein fortlaufender Prozess ist, hat die Arbeit von Mauss unser Interesse, weil sie Gesellschaftsformen zeigt, die dieses Bedürfnis mittels periodisch ändernden Zugehörigkeiten und einem abwechselnd individualistischen Lebensstil mit einem intensiven Gruppenleben aufnehmen. Es ist für mich denkbar, dass sich die in der Einleitung angesprochene Überforderung dann einstellt, wenn der psychische Aspekt, den José Bleger (1967) «synkretische Sozialität» nennt, sich dieser gesellschaftlichen "Anverwandlung» entzieht und ihr damit erst recht in äusserster Schärfe unterworfen ist. Aus dieser Perspektive wird deutlich, dass ich nicht den Aspekt der Masse betrachte, den Freud als «kollektive Intelligenzhemmung» (1974, S. 80) bezeichnet hat. Ich wende mich einem psychischen Funktionieren zu, das sowohl in dualen wie in gruppalen Situationen an der Arbeit der Darstellbarkeit und Symbolisierung notwendigerweise beteiligt ist. Im Zusammenhang mit der Betrachtung der Arbeit von Mauss als einem Modell von sozialer Mobilität, ist es mir wichtig, nochmals den dialektischen Aspekt einer Reversibilität von Progression und Regression zwischen PS $\Leftrightarrow$ D hervorzuheben. Ich meine, dass in einem Sektor der Psyche die unbewusste Oszillation von Chaos zu Struktur, von Desintegration zu Reintegration, von Zersplitterung und Spaltung zu Kohärenz, ständig am neuen Entstehen von Symbolisierungen beteiligt ist. Deshalb drängt sich eine andere Sicht auf Identität und Entwicklung als aus der des Phasenmodells auf.

Als schöne Illustration der ständigen Wirksamkeit von $P S \Leftrightarrow D \Leftrightarrow P S \Leftrightarrow D$ möchte ich den LeserInnen die folgende Textstelle, die ich im Verlauf der Arbeit am Thema «Ideologie der Sesshaftigkeit und Mobilität» gefunden habe, nicht vorenthalten. «Dans la pensée inuit, le sexe est conçu comme instable, aussi bien lorsqu'il s'agit du mythe d'origine des premiers humains qu'à l'occasion de chaque naissance. Un sexe peut toujours se transformer en l'autre sexe. [...] Dans les années 
1990, j'ai recueilli de nouveaux témoignages sur le chamanisme et sur l'androgynie qui semblait souvent lui être associée. Et là, s'est confirmé le dualisme saisonnier relevé par Mauss. Les grands rituels chamaniques étaient bien pratiqués l'hiver, lors des pleines lunes, au cours de la nuit polaire. Mais, contrairement à ce que pensait Mauss, ce n'est pas le dualisme saisonnier qui leur donne sens, c'est le dualisme des sexes, qui sert à penser toutes les autres grandes différenciations (nuit/ jour, hiver/été, mer/terre, lune/soleil...). Et, par surcroît, à cette division duelle du cosmos, s'ajoutait un troisième terme, un troisième sexe (ou genre), celui de l'androgynie, qui servait à exprimer la médiation. J'ai trouvé dans les manuscrits de Svend Frederiksen la confirmation de cette hypothèse que j'avais formulée dès 1986 » (d'Anglure, 2004, S. 124).

Und weil das obige Zitat so direkt zu uns Psychoanalytikern spricht, dass sich eine Deutung erübrigt, ganz zum Schluss noch ein Blick auf einen anderen Aspekt der aktuellen Situation der Inuit. Ihre ständige physische Mobilität ist heute nicht dem jahreszeitlichen Wandel geschuldet, sondern eine Konsequenz der Konzentration von einer grossen Zahl von früher nomadisierenden Familien am selben Ort. So ist die erzwungene Immobilität als Resultat der Einführung eines sesshaften Lebensstils ein moderner «stress agent». Früher war Mobilität ein zentraler Bestandteil von Konfliktlösung (Condon, 1982, S. 157). Das heisst, heute müssen die Inuit aus den Siedlungen «flüchten», weil es ihnen zu eng wird. Wenn Sahlins einmal fand "Mobility and property are in contradiction" (1976, S. 12), muss dies für die modernen Inuit korrigiert werden. Heute gibt es Inuit, die Flugzeuge besitzen, die es ihnen erlauben, den Subsistenzradius der Karibujagd zu erweitern und Besuche in anderen Siedlungen zu machen (Sahlins, 1999, XV). MitVerblüffung kann man feststellen, dass «traditionelle» Kulturen nicht völlig inkompatibel mit dem «Spätkapitalismus» sind (ebd.). Auch hier hat eine statische Vorstellung von Kultur für die «Überraschung» gesorgt.

\section{Literatur}

Agamben, G. (1995). Homo sacer. Il potere sovrano e la nuda vita. Torino: Giulio Einaudi editore.

Baranger, M. \& Baranger, W. (1960-1961). La situación analítica como campo dinámico Revista Uruguaya de Psicoanálisis, $I V, 1$.

Bazzi, D. (2012). Bollwerk. Eintrag im Lexikon der Raumphilosophie. S. Günzel (Hrsg.) S. 61. WBG Darmstadt.

Bion, W. R. (1971). Erfahrungen in Gruppen und andere Schriften. Stuttgart: Ernst Klett Verlag. [1961] 
Bion, W. R. (1984). Attention and Interpretation. London: Karnac. [1970]

Bion, W. R. (1994) Cogitations. New Extended Edition. London: Karnac.

Bion Talamo, P. (2011). Mappe per l'esplorazione psicoanalitica. A cura die Anna

Baruzzi Prefazione di Claudio Neri. Roma: Borla.

Bleger, J. (1978, 1967). Simbiosis y ambigüedad. Estudio Psicoanalítico. Buenos Aires: Editorial Paidos.

Briggs, J. L. (1970). Never in Anger. Portrait of an Eskimo Family. Cambridge: Harvard University Press.

Condon, R. G. (1982). Seasonal Variation and Interpersonal Conflict in the Central Canadian Arctic. Ethnology, XXI(2), 151-164.

Duby, G. (1985). Ritter, Frau und Priester. Die Ehe im feudalen Frankreich. Frankfurt a. M.: Suhrkamp.

D’Anglure, B. S. (2004) Mauss et l'anthropologie des Inuit. Sociologie et sociétés, 36(2), 91-130. URI: http://id.erudit.org/iderudit/011050ar, konsultiert am 18.4.2013.

Ferro, A. (2013). Modello onirico della mente. In A. Ferro (Hrsg.) Psicoanalisi oggi (S. 175-220). Roma: Carocci editore.

Freud, S. (1974). Massenpsychologie und Ich-Analyse. Studienausgabe Bd. IX. Frankfurt a. M.: S. Fischer Verlag. [1921]

Freud, S. (1974). Totem und Tabu (Einige Übereinstimmungen im Seelenleben der Wilden und der Neurotiker) [1912-13] Studienausgabe Bd. IX. Frankfurt a. M.: S. Fischer Verlag.

Grotstein, J. S. (2007). A Beam of Intense Darkness. Wilfred Bion's Legacy to Psychoanalysis. London: Karnac.

Klein, M. (1975). A Contribution to the Psychogenesis of Manic-Depressive States. In M. Klein, Love, Guilt an d Reparation \& Other Works 1921-1945, (S. 262298). New York: Dell Publishing Co. [1935]

Klein, M. (1946). Notes on Some Schizoid Mechanisms. Journal of Psychotherapy Practice and Research, 5(2), 164-179.

Lantis, M. (1950). The Religion of the Eskimos. In V. Ferm (Hrsg.), Forgotten Religions. Including some living Primitive Religions (S. 311-339). New York. Mauss, M. (1978). Über den jahreszeitlichen Wandel der Eskimogesellschaften. Soziologie und Anthropologie. Bd. 1. München: Carl Hanser Verlag. [1950] Meillassoux, C. (1975). Femmes, greniers et capitaux. Paris: Maspero.

Neri, C. (1985). Osservazioni a proposito della analizzabilità nella situazione di gruppo. Gruppo e Funzione Analitica, VI, 19-24. 
Neri, C. (2006). Gruppenprozesse. Theorie und Praxis der psychoanalytischen Gruppentheorie. Giessen: Psychosozial-Verlag.

Pinson, D. (2012). De l'ancrage aux voyages: retour sur la morphologie sociale de Marcel Mauss ou comment comprendre l'espace en le représentant. In Rhuthmos. [en ligne]. http://rhuthmos.eu/spip.php?article, S. 770, konsultiert am 1.4.2013.

Sahlins, M. (1976). Stone Age Economics. London. Tavistock Publications.

Sahlins, M. (1999). What is Anthropological Enlightenment? Some Lessons of the Twentieth Century. Annual Reviews of Anthropology, 28:i-xxiii.

Torres, N. und Hinshelwood R. D. (Hrsg.) (2013). Bion's Sources. The shaping of his Paradigms. New York: Routledge. 